Revista Brasileira de Cartografia

ISSN 1808-0936 | https://doi.org/10.14393/revbrascartogr

Sociedade Brasileira de Cartografia, Geodésia, Fotogrametria e Sensoriamento Remoto

\title{
Dispersão e fragmentação urbana: uma análise espacial com base na distribuição da população
}

\section{Urban sprawl and fragmentation: a spatial analysis based on population distribution}

\author{
José Diego Gobbo Alves ${ }^{1}$ e Álvaro de Oliveira D’Antona ${ }^{2}$
}

1 Universidade Estadual de Campinas, Núcleo de Estudos e Pesquisas Ambientais, Campinas, Brasil. jdgobboalves@gmail.com. ORCID: https://orcid.org/0000-0002-4185-1579

2 Universidade Estadual de Campinas, Faculdade de Ciências Aplicadas, Limeira, Brasil. adantona@unicamp.br.

ORCID: https://orcid.org/0000-0003-1710-6277

Resumo: Pesquisas sobre a dispersão e a fragmentação urbana consideram indicadores não-espaciais como a densidade demográfica e a centralidade econômica em suas análises. De forma alternativa, o propósito do artigo é apresentar uma metodologia baseada em métricas da distribuição espacial da população em uma grade estatística regular, potencializando o uso de dados demográficos. Em um Sistema de Informação Geográfica, foram calculados indicadores com dados do Censo Demográfico de 2010 para os 23 municípios da Aglomeração Urbana de Piracicaba (AUP), estado de São Paulo, de tal modo a avaliar a forma urbana, a extensão e a fragmentação da mancha urbana em cada município. Os resultados mostram que os municípios apresentam características como fragmentação e espraiamento em intensidades diferentes, e que a distribuição espacial observada na AUP se orienta conforme vetores de ocupação territorial, como as rodovias. Detectaram-se correlações positivas e fortes entre volume da população, dimensões e formas da mancha urbana. O caso da AUP indica que a metodologia é propícia para a leitura espacial dos fenômenos considerados e para estudos comparativos. Com dados de fácil acesso, a técnica oferece boa resolução espacial, podendo ser replicada para outros recortes. Os resultados permitem dialogar com a produção bibliográfica sobre a dispersão urbana, seja pela explicitação do seu componente espacial, seja pela possibilidade de interação com outros indicadores não-espaciais.

Palavras-chave: Distribuição espacial da população. Aglomeração Urbana de Piracicaba. Indicadores espaciais.

\begin{abstract}
Research on dispersion and urban fragmentation considers non-spatial indicators such as demographic density and economic centrality in their analyses. Alternatively, the purpose of the article is to present a methodology based on metrics of the spatial distribution of the population in a regular statistical grid, enhancing the use of demographic data. In a Geographic Information System, indicators with data from the demographic census of 2010 is calculated for the 23 municipalities' Urban Agglomeration of Piracicaba (AUP), state of São Paulo, in such a way as to evaluate the urban form, extent and fragmentation of the urban area in each municipality. The results show that the municipalities have characteristics such as fragmentation and spreading in different intensities and that the spatial distribution observed in the AUP is guided according to a territorial occupation vectors, such as highways. The positive and strong correlations between volume, population and forms of the urban area were detected. The case of the AUP indicates that the methodology is propitious for the spatial reading of the phenomena considered and for comparative studies. With easy-to-access data, the technique offers good spatial resolution and can be replicated to other clippings. The results allow dialoguing with the bibliographic production about urban sprawl, either by explaining the spatial component, or by the possibility of interaction with other non-spatial indicators.
\end{abstract}

Keywords: Spatial distribution of the population. Urban Agglomeration of Piracicaba. Spatial indicators.

\section{INTRODUÇÃO}

A dispersão urbana é um fenômeno contemporâneo reflexo do crescimento espacial desordenado das cidades, atrelado aos estilos de vida moderno de segregação e autossegregação, deixando marcas sócio- 
espaciais das desigualdades (REIS FILHO, 2006; LIMONAD, 2007; SPOSITO, 2009; NASCIMENTO JUNIOR, 2017). A dispersão e a fragmentação urbana, expressão espacial de um modo de vida urbano, devem ser consideradas dentre as características das formas das cidades. Suas medidas permitem avaliar se as cidades são territorialmente compactas ou espraiadas, contínuas ou descontínuas, com ou sem fragmentos e com variado grau de conectividade espacial (SPOSITO, 2009), sendo importantes para a compreensão do fenômeno e para a elaboração de políticas públicas em âmbitos municipais e regionais.

A literatura aponta para a importância da utilização de diversos indicadores para a mensuração da dispersão urbana em uma cidade, de tal modo a permitir a comparação em escalas regional e local (GALSTER et al., 2001, REIS FILHO, 2006, OJIMA, 2007; GONÇALVES, 2011; GONÇALVES; KRAFTA, 2016). Apesar da pluralidade de propostas metodológicas, nem todos os estudos mensuram espacialmente a dispersão e a fragmentação urbana.

O pioneiro estudo de Galster et al. (2001) sugere que os indicadores para a avaliação da dispersão urbana são: densidade residencial, continuidade da área disponível para urbanização, grau de concentração da área urbana, grau de agrupamento da área urbana, relação das áreas (residenciais ou não) próximas à centros de negócios, número de núcleos em uma área urbana, diversidade no uso do solo e proximidade entre esses diferentes usos de solo. Trata-se de um trabalho relevante para os estudos da dispersão urbana posto que os autores rompem com a tradição de utilizar apenas a densidade demográfica (GONÇALVES, 2011). Já Ewing et al. (2002) elaboraram um conjunto de 22 indicadores para classificar a dispersão urbana, sendo densidade demográfica, uso do solo, concentração de atividades, forma e extensão das quadras, alguns exemplos das medidas utilizadas.

Como referência teórica brasileira, destacam-se os estudos de Reis Filho (2006) e Ojima (2007). A análise do processo de dispersão urbana paulista realizadas por Reis Filho (2006) serviu de base para outros trabalhos como o de Schutzer (2012), Trentin (2012), Nandin (2013) e Eigenheer e Somekh (2017). O trabalho de Ojima (2007) traz uma análise da dispersão urbana em caráter regional, considerando dimensões espaciais e não espaciais, com os seguintes indicadores: densidade demográfica total de uma cidade; o índice de vizinhança próxima; orientação e linearidade (orientação que as áreas urbanas seguem); integração e centralidade.

Posteriormente, Gonçalves (2011) e Gonçalves e Krafta (2016) trazem um conjunto das principais metodologias utilizadas para a mensuração da dispersão urbana. Tais metodologias incluem dimensões espaciais (forma, fragmentação, compacidade, acessibilidade, entre outros) e dimensões não-espaciais (características sociais e demográficas da população e tipos de uso do solo), geralmente atreladas à escala municipal de análise.

Para identificar padrões de ocupação territorial, sobretudo no Brasil, os estudos utilizam setores censitários, imagens de satélites e tipologia das quadras e das ruas. Contudo, mesmo nos casos em que se mede a distribuição espacial da população, raros são os estudos que utilizam unidades territoriais de análise regulares (com mesmo formato e dimensões), tais como as células de uma grade estatística. Particularmente relevante ao caso brasileiro, a grade estatística criada pelo Instituto Brasileiro de Geografia e Estatística (IBGE) para a divulgação dos dados do Censo Demográfico de 2010 tem um potencial analítico ainda pouco explorado (BUENO, 2014; IBGE, 2016). As grades regulares favorecem a aplicação de estatísticas espaciais e tendem a ser mais eficazes em análises de padrões espaciais de distribuição da população, principalmente pela padronização das suas células e pela melhor resolução espacial, comparativamente a outras unidades territoriais de análise (D’ANTONA; BUENO; DAGNINO, 2013; BUENO, 2014).

Dentre os trabalhos que utilizam grades regulares para analisar padrões de distribuição espacial da população, destacam-se os de D'Antona e Bueno (2015a, 2015b), Iwama et al. (2016), Côrtes (2017) e Pedro e Queiroz-Filho (2017). Estudos mais recentes lançam mão de metodologias baseadas em grades regulares para análise de fenômenos socio-espaciais, buscando modelar o crescimento populacional nos próximos anos (ZHOU; VARQUEZ; KANDA, 2019); visando identificar o grau de dispersão e densidade da distribuição espacial da população (AURAMBOUT; BARRANCO; LAVALLE, 2018); ou as possíveis mudanças nas características demográficas e econômicas da população (LLOYD et al., 2017).

Contudo, faltam estudos que mensurem e explorem, além do grau de dispersão urbana, a forma da 
distribuição da população no espaço. O propósito de nosso artigo é apresentar uma metodologia composta por um conjunto de métricas espaciais calculadas com base na distribuição espacial da população para mensurar e comparar a dispersão e a fragmentação urbana, utilizando uma grade estatística oficial, de amplo acesso. Explora-se, portanto, as potencialidades do uso de dados comumente utilizados em estudos demográficos para a análise de cunho espacial do fenômeno da dispersão urbana.

A aplicação ao caso dos 23 municípios membros da Aglomeração Urbana de Piracicaba (AUP), ente regional localizado na macrometrópole paulista (EMPLASA, 2018), se motiva pelas características do objeto de estudo e pelo o que ele pode revelar sobre o fenômeno da dispersão urbana. A AUP é composta por cidades de porte pequeno e médio e, portanto, possibilita identificar a expressão material da dispersão urbana em duas tipologias de cidades distintas, sobretudo nas cidades de pequeno porte, ainda pouco exploradas.

\section{O FENÔMENO DA DISPERSÃO URBANA}

Há uma pluralidade de estudos de casos que buscam identificar as causalidades do fenômeno da dispersão urbana. De modo geral, a dimensão espacial da dispersão urbana está associada à uma baixa densidade populacional, baixa conectividade entre os fragmentos de mancha urbana e alto custos e uso de energia e transportes (AURAMBOUT; BARRANCO; LAVALLE, 2018). Apesar da característica espraiada e, geralmente, descontínua que o fenômeno apresenta, os fatores condicionantes desses processos possuem particularidades de acordo com os contextos sócio-espaciais.

Nos Estados Unidos, o processo de dispersão urbana data a partir da segunda metade do século XX com o crescimento espraiado das cidades (urban sprawl), o qual foi impulsionado pela abertura de vias de transporte automobilísticos, pelo uso de automóveis individuais e a criação de Shopping Centers próximos aos novos fragmentos da mancha urbana, os conhecidos subúrbios da classe média estadunidense (MANCINI, 2008). Como exemplo, a cidade de Los Angeles, entre 1970 e 1990, apresentou uma expansão urbana de 300\% enquanto a população cresceu apenas 45\% (MEADOWS, 1999).

$\mathrm{Na}$ China, as áreas industriais exercem papel central dentre os indutores da dispersão urbana pois são criadas de forma descontinua em relação a cidade construída. O Estado é o principal agente promotor da dispersão, primeiro pelos incentivos fiscais às indústrias e, segundo, pela política de financiamento de terras. A reforma tributária da China centralizou nacionalmente a captação de uma série de impostos, diminuiu as receitas locais e, como saída para uma maior captação de recursos, os governos locais viram o financiamento de terras como um mecanismo para aumentar a arrecadação, o que estimula a expansão territorial (LIU et al., 2018).

Apesar das cidades latino-americanas seguirem a tendência estadunidense de urbanização, isto é, o caráter espraiado de ocupação, os determinantes do crescimento espacial das cidades latino-americanas são distintos. Enquanto nas cidades estadunidenses a dispersão urbana foi impulsionada por um novo padrão de consumo espacial e como uma fuga dos grandes centros urbanos deteriorados, nas cidades latino-americanas a pobreza dos grupos sociais desfavorecidos economicamente e a prosperidade das elites aparecem como determinantes iniciais da dispersão urbana (MANCINI, 2008).

No Brasil, a dispersão urbana não é típica apenas das metrópoles. O fenômeno é verificado em cidades médias e pequenas em todos os estados brasileiros. As rodovias possuem papel central para viabilização desse processo e, consequentemente, da regionalização do cotidiano (REIS FILHO, 2006; TRENTIN, 2012). Isso ocorre pois as rodovias viabilizam morar cada vez mais distante da área central da cidade, permitindo também a locomoção intra-regional da população para a realização das atividades do cotidiano. Dessa forma, o mercado imobiliário busca criar novos condomínios residenciais às margens das rodovias, tornando-as grandes avenidas que drenam o fluxo de automóveis (CUNHA et al., 2013).

A AUP, apesar de presente na macrometrópole paulista (EMPLASA, 2008), possui dinâmicas sócioespaciais distintas das regiões metropolitanas de Campinas e de São Paulo. Contudo, no que tange ao processo de urbanização do território, observa-se semelhanças no padrão espraiado de produção espacial, sobretudo pela a construção de loteamentos distantes da mancha urbana consolidada, mas próximos de rodovias (TRENTIN, 2012). Tal quadro demanda a aplicação de indicadores espaciais que possibilitem estabelecer, quantitativamente, comparações futuras com outras regiões paulistas. 


\section{MATERIAIS E MÉTODOS}

A Aglomeração Urbana de Piracicaba (AUP) é uma unidade regional que foi institucionalizada pela Lei Estadual $\mathrm{N}^{\circ}$ 1.178, de 26 de junho de 2012 (SÃO PAULO, 2012). Atualmente, é constituída por 23 municípios, sendo eles: Águas de São Pedro, Analândia, Araras, Capivari, Charqueada, Conchal, Cordeirópolis, Corumbataí, Elias Fausto, Ipeúna, Iracemápolis, Laranjal Paulista, Leme, Limeira, Mombuca, Piracicaba, Rafard, Rio Claro, Rio das Pedras, Saltinho, Santa Gertrudes, Santa Maria da Serra e São Pedro (Figura 1). Ressalta-se que o município de Laranjal Paulista foi incluído posteriormente, no ano de 2013.

A AUP concentra cerca de 1,4 milhão de habitantes, o que representa 3,3\% da população de todo o estado de São Paulo (EMPLASA, 2017). Todos os municípios membros apresentam um grau de urbanização acima de 80\%, com a predominância de municípios com um grau de urbanização acima de $90 \%$ (EMPLASA, 2018), o que instiga analisar quais são os padrões de distribuição espacial da população.

Figura 1 - Localização da Aglomeração Urbana de Piracicaba - SP.

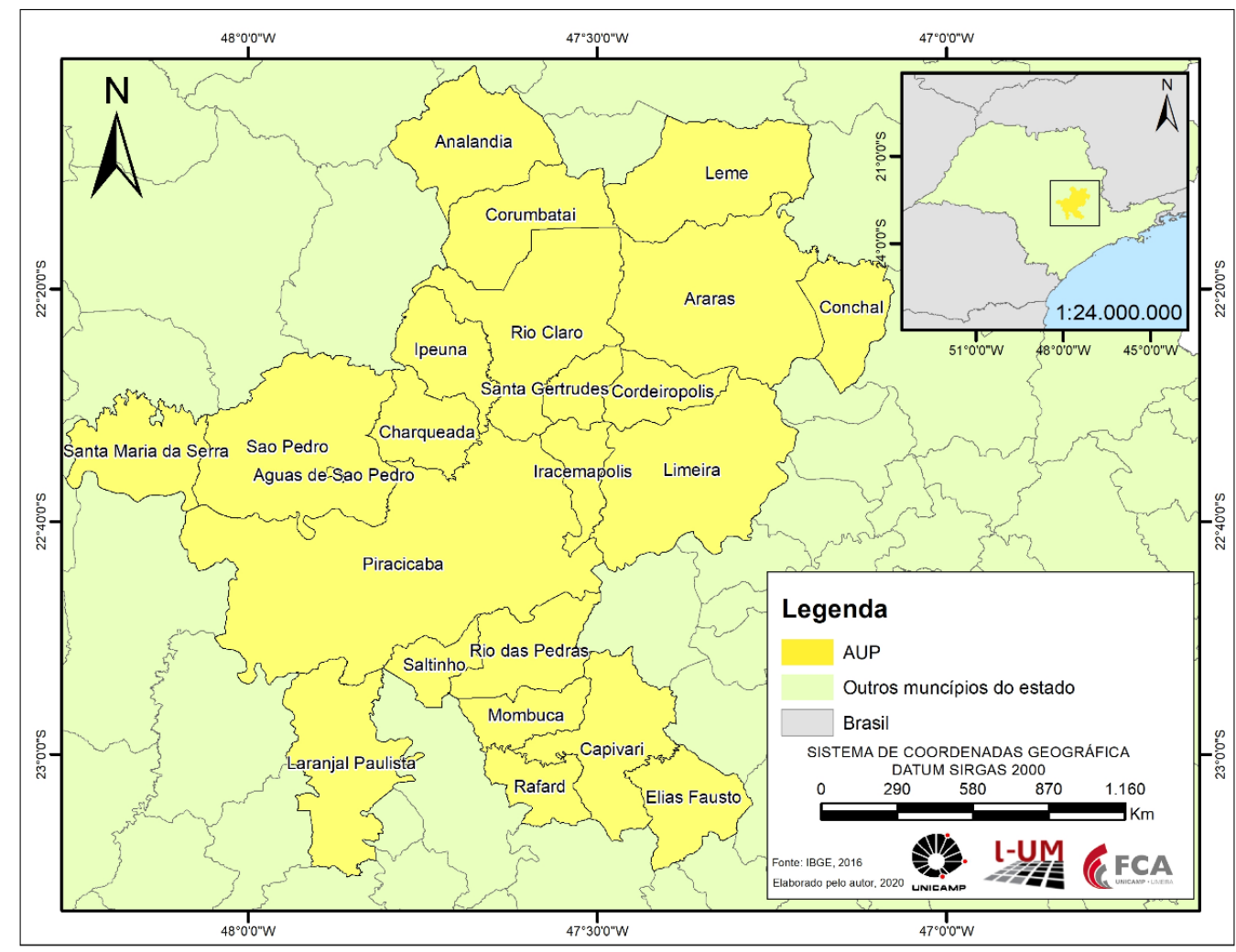

Fonte: Os autores (2020).

Para o estudo, a população residente da AUP foi distribuída conforme a Grade Estatística oficial do Censo Demográfico 2010 (IBGE, 2016). A grade consiste em um sistema regular de células com medida lateral de 37,5 arcos segundos em áreas rurais e células com 7,5 arcos segundos em áreas urbanas, o que corresponde a aproximadamente $200 \mathrm{~m}$ x $200 \mathrm{~m}$ para as áreas urbanas e $1 \mathrm{~km} \mathrm{x} 1 \mathrm{~km}$ para as áreas rurais (BUENO, 2014; D'ANTONA; BUENO, 2015b; IBGE, 2016). Tais dimensões oferecem uma resolução espacial superior àquela oferecida pelos setores censitários, sobretudo nas áreas rurais e periurbanas, e a regularidade das células favorece as aplicações de análises estatísticas.

Além da população residente, cada célula da grade estatística agrega as variáveis de número de domicílios, sexo dos residentes e a situação (rural ou urbana) da célula. Apesar do número limitado de variáveis em relação ao universo do Censo Demográfico, uma vantagem do uso da grade é que a distribuição da população se baseia na localização (coordenadas) dos domicílios recenseados e não em algoritmos - os quais somente foram aplicados pelo IBGE nos casos de omissão da localização dos domicílios.

O uso da célula como unidade territorial de análise representa um avanço para as análises espaciais em relação ao uso do setor censitário, unidade operacional de coleta. Por suas diminutas dimensões, as células 
oferecem melhor resolução espacial da distribuição da população e, consequentemente, melhor aderência aos recortes estudados (BUENO, 2014). Entretanto, a eficácia no uso em diferentes recortes espaciais varia de acordo com o objeto de estudo, pois, como mostrado por Pedro e Queiroz-Filho (2017), as dimensões das células urbanas não ofereceram um significativo avanço para a análise de aglomerados subnormais, comparativamente aos setores censitários analisados, os quais possuem um maior potencial para a análise das áreas de favela como um todo.

Entre outras vantagens do uso da grade estatística para a análise espacial, incluem-se a adaptação a recortes espaciais; a hierarquia; a flexibilidade; a versatilidade e a estabilidade espaço-temporal, ou seja, a dimensão física das células que não é alterada de um censo para o outro, como ocorre com os setores censitários e os limites políticos administrativos, permitindo análises comparativas (BUENO; D'ANTONA, 2012, 2014; BUENO, 2014; IBGE, 2016). Apesar desse estudo não ser longitudinal, a partir da divulgação dos dados do próximo Censo Demográfico será possível a realização de futuros estudos comparativos.

Para a montagem da grade da área de estudo, foram utilizadas as folhas 25 e 35 presentes no repositório de dados do IBGE (IBGE, 2016). Em um Sistema de Informação Geográfica (SIG), por meio do software Arcgis, versão 10.4, foram selecionadas apenas as células que compreendem os limites municipais dos 23 municípios da AUP. Utilizando a grade estatística da AUP como base, foram calculados os indicadores dos componentes espaciais da dispersão urbana em cada município. Para os cálculos, foi considerada a população residente nas áreas correspondentes às células urbanas - aquelas situadas em setores censitários urbanos. Ou seja, foram utilizadas apenas as células urbanas com população residente maior que zero.

Para medir a concentração da forma urbana, foi utilizado o indicador DMC - Distância Média entre as Células (no ArcGis, Standard Distance). Tal medida utiliza as feições geográficas (células) como base para a criação de um polígono circular resultante da análise automática da disposição destas feições, permitindo avaliar o quanto a população está concentrada em células em torno do centro médio geométrico de cada área urbana analisada (Figura 2).

Para medir a forma urbana foi utilizado o indicador DDC - Distribuição Direcional das Células (no ArcGis, Directional Distribution; Standard Deviational Ellipse), levando em conta a excentricidade da elipse que agrega as células com maior população residente em cada município, a partir do cálculo da excentricidade da forma gerada pelo indicador (Figura 2). O DDC se baseia na disposição das feições (células) em torno de um centro médio, indicando se a forma urbana segue um padrão radial ou elíptico, desse modo foi calculado a excentricidade das formas, para identificar o quão afilada são as mesmas. O DMC e o DDC permitem perceber evidências da expansão urbana a partir de um centro e se há influências de eixos de ocupação (por exemplo, cursos d'água e vias terrestres).

Figura 2 - Medidas espaciais do ArcGis: Distância Média entre as Células e Distribuição Direcional das Células.

Distância média entre as células

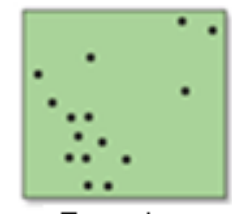

Entrada

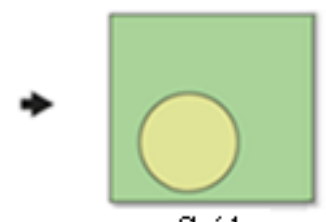

Saída

\section{Distribuição Direcional das células}
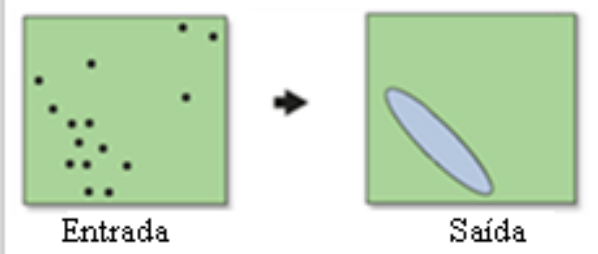

Saida

Fonte: Adaptada de Esri (2019a; 2019b).

Métricas de ecologia da paisagem foram utilizadas para medir a fragmentação da mancha urbana em cada um dos municípios. Tais métricas permitem identificar e analisar a estrutura da paisagem, isto é, padrões espaciais passíveis de serem observados nas distintas formas de ocupação espacial da população. Estudos recentes já utilizam métricas de ecologia da paisagem para a análise urbana, tais como o de Coelho (2015; 2016), abrindo um campo de estudos comparativos entre o meio urbano e o meio rural, utilizando as mesmas métricas de análise, como já realizado nos estudos de Esbah et al. (2012), Tagil, Gormus e Cengiz (2018), entre outros.

Para o cálculo, as células urbanas com população residente foram dissolvidas para formar um 
continuum de mancha urbana. Ou seja, as células urbanas contíguas com população residente foram agregadas, constituindo novos polígonos com os fragmentos de manchas urbanas de cada município. Salienta-se que o termo mancha urbana empregado nesse trabalho se refere ao conjunto de células urbanas com população residente, a partir da caracterização do IBGE. Foram calculados os seguintes indicadores das manchas urbanas municipais: a) Área Total da Mancha Urbana; b) Número de Fragmentos da Mancha Urbana; c) Tamanho Médio dos Fragmentos da Mancha Urbana e d) Indicador Médio Ponderado pela Área (IMPA). Respectivamente, os indicadores apresentam a área total da mancha urbana $\left(\mathrm{em} \mathrm{m}^{2}\right)$; a quantidade de fragmentos presentes em cada mancha urbana e o tamanho médio dos fragmentos por município $\left(\mathrm{em}^{2}\right)$ e, por fim, o indicador médio da forma ponderado pela área expressa o quanto a mancha é próxima de um círculo e contribui na reflexão mostrando se a disposição dos fragmentos da mancha urbana tende a ser concêntrica ou não. Quanto mais próxima de 1, mais a mancha estará próxima de um círculo.

Os dois conjuntos de indicadores - os de concentração da forma urbana e os de fragmentação da mancha urbana - oferecem uma leitura integrada e comparativa do componente espacial da dispersão urbana. Eles permitem a leitura do espaço em unidades geográficas padronizadas, e através de indicadores efetivamente espaciais. Buscando estabelecer conexões entre as variáveis com o propósito de encontrar padrões de formafragmentação da mancha urbana, foi realizado teste de correlações estatísticas lineares (Pearson) entre as variáveis empregadas

\section{RESULTADOS}

O mapa com a distribuição espacial da população na AUP (Figura 3) oferece uma visão geral da distribuição das células ocupadas. Nas células rurais - aquelas com $1 \mathrm{~km}$ de lado - se verifica uma ocupação por grandes extensões em toda a área de estudo, mas com baixa densidade por célula (até 100 habitantes por célula). Nas células urbanas - aquelas com $200 \mathrm{~m}$ de lado - se verifica ocupação mais adensada. As células urbanas com população se concentram em porções relativamente bem delimitadas em cada município, geralmente sem contiguidade entre municípios. Exceto Capivari e Rafard, e de Rio Claro e Santa Gertrudes, os quais compartilham áreas urbanas conurbadas, os outros compartilhamentos são de caráter rural. Essa ocupação abre um leque de possibilidades de pesquisa sobre o impacto dos limites políticos administrativos para a continuação e identificação territorial da população e de seus fluxos.

Figura 3 - Distribuição da população rural e urbana na Aglomeração Urbana de Piracicaba, 2010.

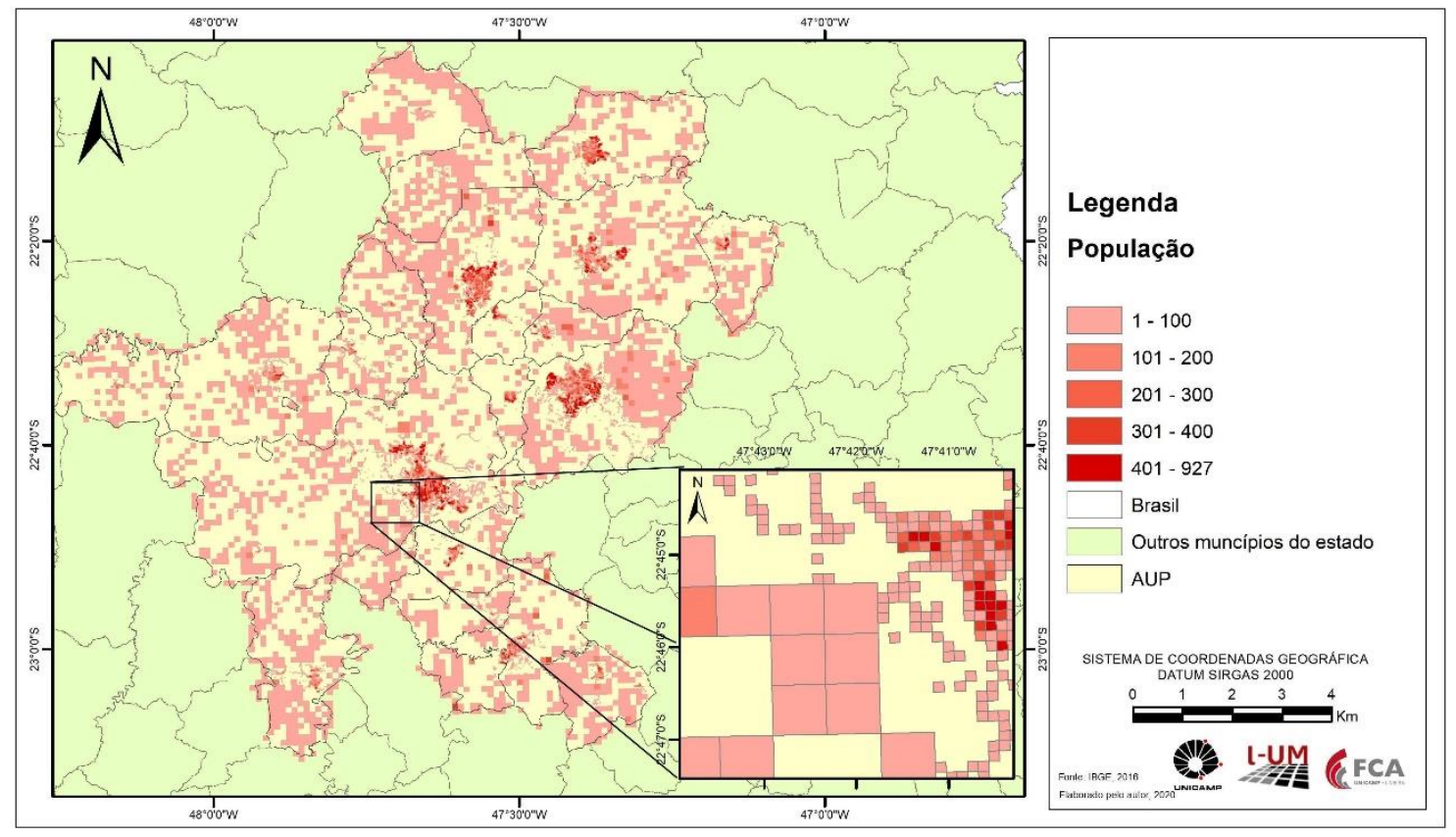

Fonte: Os autores (2020).

A melhor resolução espacial possibilita essa leitura mais detalhada da distribuição da população, 
diferentemente do que ocorre com o uso dos setores censitários, sobretudo em relação à identificação de porções sem residentes. Do total de 6.471 células rurais, 2.560 células $(39,6 \%)$ apresentam população residente diferente de zero, perfazendo um total de 45.681 habitantes em uma área rural ocupada de aproximadamente de $2.560 .000 \mathrm{~m}^{2}$. Do total de 33.602 células urbanas, 10.643 células (32\%) apresentam população residente, perfazendo um total de 1.272.366 habitantes em uma área urbana ocupada de aproximadamente $425.720 \mathrm{~m}^{2}$.

Levando em conta a população e a área municipal por células segundo sua situação rural ou urbana, o significado espacial do elevado grau de urbanização dos municípios se evidencia: 96,5\% da população reside em 16,63\% da área total da AUP, em áreas urbanas ocupadas; 3,5\% da população reside em 83,67\% da área total da AUP, em áreas rurais ocupadas. Por outro lado, ainda que o maior contingente populacional esteja em células urbanas, e que as áreas urbanas sejam menos extensas que as rurais na maior parte dos municípios da AUP, há relativamente um número baixo de células urbanas ocupadas, como pode ser observado na Tabela 1 com base nos dados do IBGE (2016).

Tabela 1 - População residente e número de células urbanas ocupadas na Aglomeração Urbana de Piracicaba, 2010.

\begin{tabular}{|c|c|c|c|c|c|c|}
\hline \multirow{3}{*}{ Municípios } & \multicolumn{3}{|c|}{ Células urbanas } & \multirow{3}{*}{$\begin{array}{c}\text { População } \\
\text { Urbana } \\
\text { (hab) }\end{array}$} & \multirow{3}{*}{$\begin{array}{r}\text { População Total } \\
\text { (hab) }\end{array}$} & \multirow{3}{*}{$\begin{array}{c}\text { Grau de } \\
\text { urbanização } \\
(\text { em \%) }\end{array}$} \\
\hline & Total & Ocupad & & & & \\
\hline & (n) & (n) & $(\%)$ & & & \\
\hline Águas de São Pedro & 117 & 55 & 47,0 & 2.089 & 2.089 & 100 \\
\hline Analândia & 414 & 142 & 34,3 & 3.274 & 3.976 & 82,3 \\
\hline Araras & 1.547 & 760 & 49,1 & 112.847 & 117.728 & 95,9 \\
\hline Capivari & 1.545 & 508 & 32,9 & 43.020 & 45.624 & 94,3 \\
\hline Charqueada & 1.034 & 276 & 26,7 & 13.881 & 14.554 & 95,4 \\
\hline Conchal & 1.059 & 220 & 20,8 & 23.876 & 25.055 & 95,3 \\
\hline Cordeirópolis & 494 & 174 & 35,2 & 18.492 & 20.300 & 91,1 \\
\hline Corumbataí & 238 & 61 & 25,6 & 2.254 & 3.689 & 61,1 \\
\hline Elias Fausto & 761 & 192 & 25,2 & 12.780 & 15.131 & 84,5 \\
\hline Ipeúna & 450 & 93 & 20,7 & 5.245 & 5.976 & 87,8 \\
\hline Iracemápolis & 516 & 127 & 24,6 & 19.651 & 20.085 & 97,8 \\
\hline Laranjal Paulista & 1.185 & 311 & 26,2 & 22.812 & 25.329 & 90,1 \\
\hline Leme & 1.676 & 572 & 34,1 & 89.694 & 91.795 & 97,7 \\
\hline Limeira & 6.036 & 1.915 & 31,7 & 268.066 & 274.844 & 97,5 \\
\hline Mombuca & 300 & 71 & 23,7 & 2.809 & 3.254 & 86,3 \\
\hline Piracicaba & 8.527 & 2.919 & 34,2 & 354.255 & 360.194 & 98,4 \\
\hline Rafard & 349 & 74 & 21,2 & 8.515 & 9.666 & 88,1 \\
\hline Rio Claro & 2.903 & 1.140 & 39,3 & 185.835 & 189.637 & 98,0 \\
\hline Rio das Pedras & 2.094 & 281 & 13,4 & 27.175 & 27.954 & 97,2 \\
\hline Saltinho & 345 & 101 & 29,3 & 5.904 & 6.663 & 88,6 \\
\hline Santa Gertrudes & 679 & 129 & 19,0 & 17.603 & 17.779 & 99,0 \\
\hline Santa Maria da Serra & 300 & 61 & 20,3 & 4.848 & 5.503 & 88,1 \\
\hline São Pedro & 1.033 & 461 & 44,6 & 27.441 & 31.222 & 87,9 \\
\hline Total na AUP & 33602 & 10643 & 31,7 & 1.272 .366 & 1.318 .047 & 96,5 \\
\hline
\end{tabular}

A ocupação territorial das áreas urbanas dos municípios da AUP segue alguns padrões (Figura 4). O primeiro deles é a proximidade da ocupação espacial com vetores como as rodovias. Essa proximidade é fundamental para a viabilização desse tipo de ocupação, extremamente dependente de veículos automotivos. 
Figura 4 - Malha viária e ocupação urbana na Aglomeração Urbana de Piracicaba, 2010.

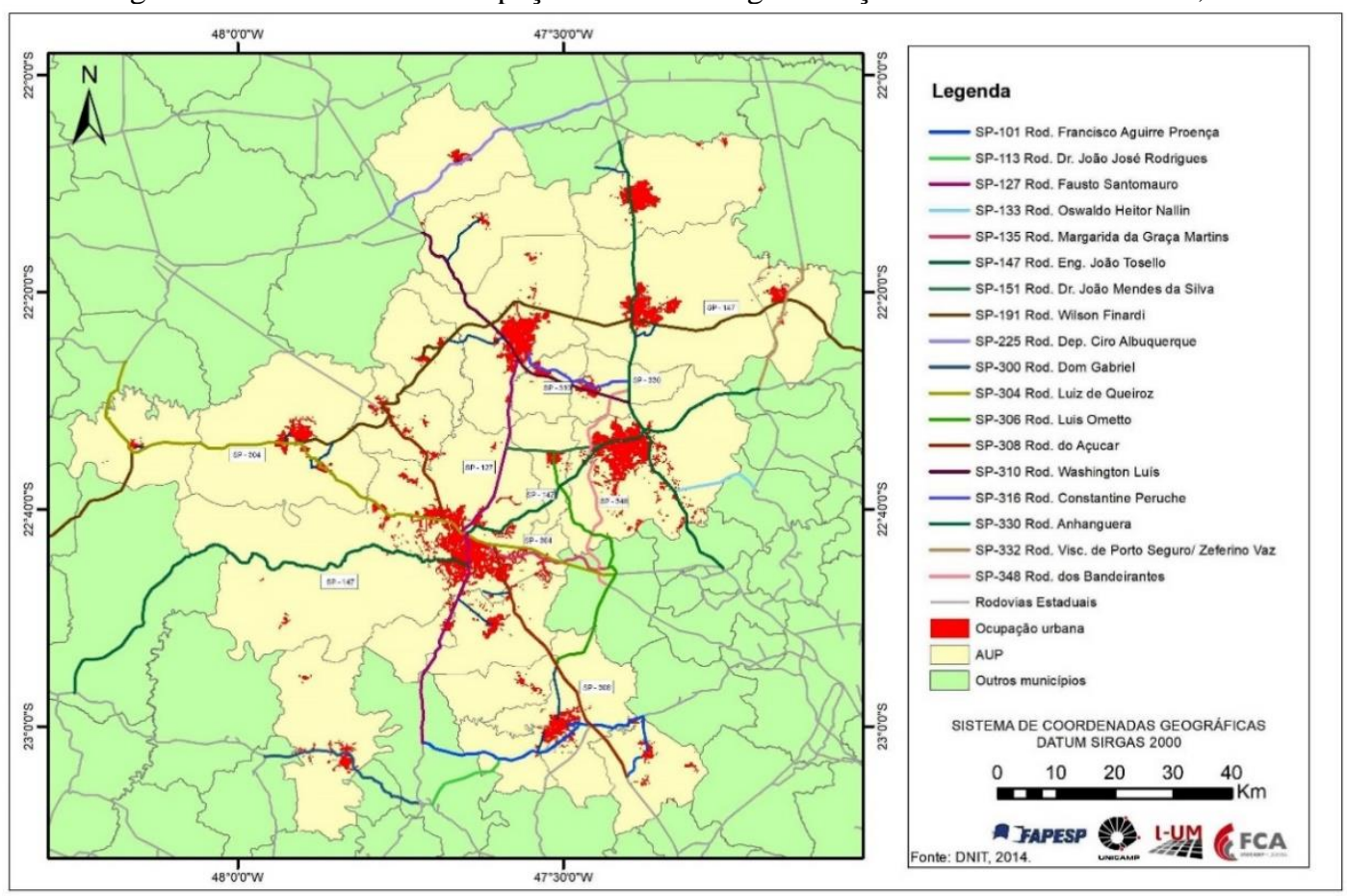

Fonte: Os autores (2020).

Os municípios de Piracicaba, Limeira e Rio Claro estão próximos de rodovias federais além das estaduais. Essa característica está vinculada à importância atual desses municípios, mas, principalmente, ao desenvolvimento histórico do interior paulista. A expansão ferroviária para o interior foi um dos fatores da ocupação de alguns municípios da região, principalmente, no caso de Limeira e de Rio Claro. Apesar de Piracicaba fazer parte dessa expansão, a ferrovia no município não foi ali tão importante como foi nos outros dois casos, bem situados em eixos centrais de interiorização (MARANDOLA JUNIOR; MAGOSSO, 2016). Tal importância é reafirmada pela expansão do modal rodoviário que seguiu e substituiu a malha ferroviária pré-existente, justamente por esta mostrar grande importância locacional.

Em relação as métricas espaciais, a Figura 5 apresenta a forma de ocupação urbana no espaço, sendo um primeiro indicativo para a análise da dispersão urbana nesses municípios. Quanto mais elíptica for a distribuição da ocupação, maior será o caráter de espraiamento longitudinal da distribuição espacial da população. No geral, observa-se que na AUP a distribuição da população é mais longitudinal do que concêntrica o que justifica a maioria das elipses serem mais afilada. É claro que barreiras físicas podem induzir um crescimento não-concêntrico da ocupação urbana, porém, não é o caso específico da topografia da região, na qual a distribuição da população está mais ligada ao aspecto rodoviário e de especulação imobiliária.

Os resultados do indicador Distância Média entre as Células (DMC), o qual dimensiona quão próximas estão as células urbanas ocupadas em relação a um centro médio, mostram que Piracicaba, Limeira, Leme e Charqueada possuem as áreas urbanas com o maior distanciamento (Tabela 2). Em contrapartida, Águas de São Pedro, Corumbataí e Iracemápolis foram o que apresentaram as maiores concentrações, ou proximidade entre as células com população urbana. Contudo, essa medida não deve ser vista isoladamente pois alguns aspectos podem fazer com que municípios que possuem caraterísticas de dispersão mais acentuadas pareçam ser menos dispersos. $\mathrm{O}$ volume da população nas células, reflexo da população municipal total, assim como o tamanho do município, podem afetar os resultados obtidos. 
Figura 5 - Representação das Métricas espaciais de distribuição urbana na Aglomeração Urbana de Piracicaba, 2010.

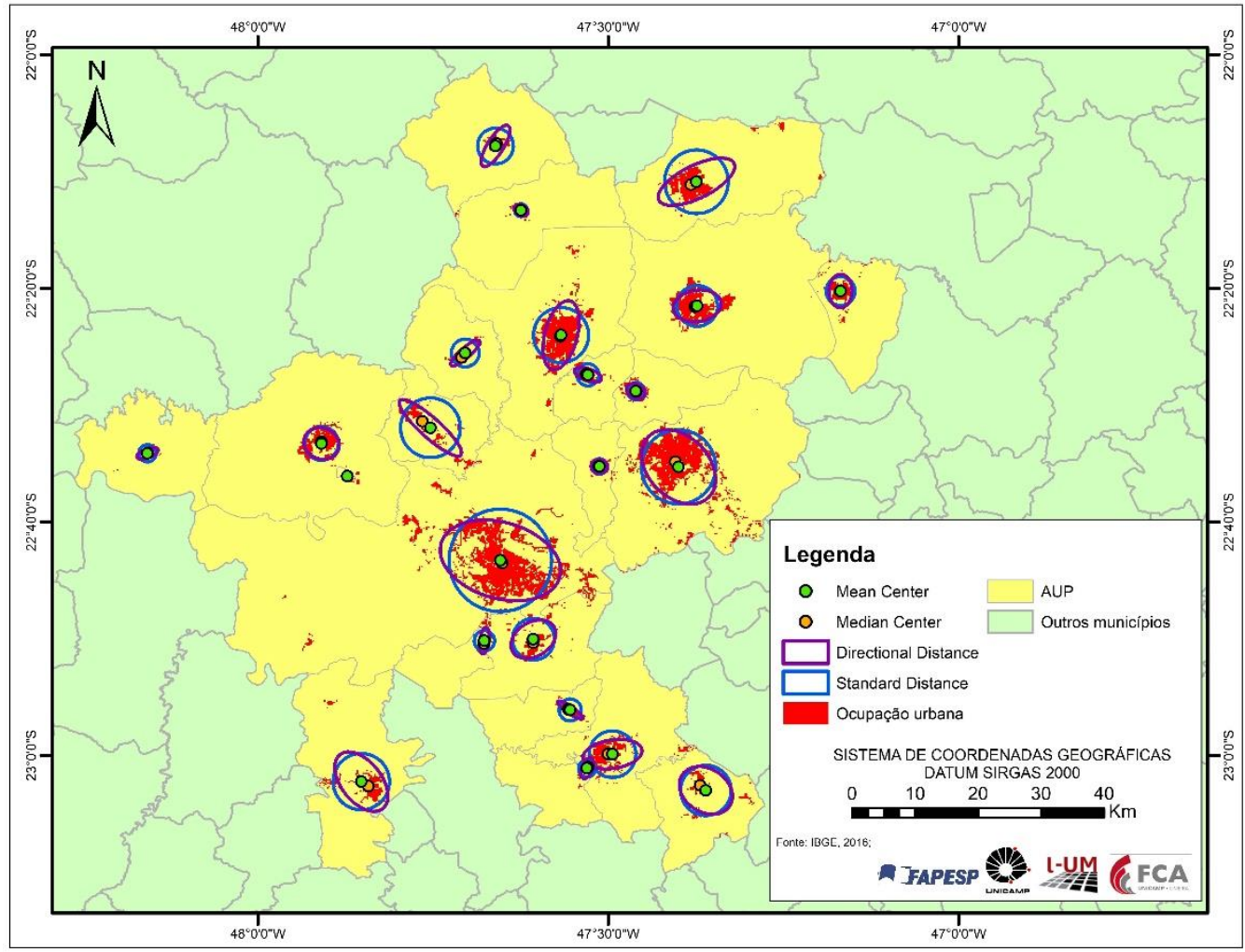

Fonte: Os autores (2020).

Tabela 2 - Distância Média entre as Células e Distribuição Direcional das Células na Aglomeração Urbana de Piracicaba, 2010.

\begin{tabular}{|c|c|c|c|c|}
\hline Municípios & Área $\left(\mathbf{m}^{2}\right)$ & $\begin{array}{c}\text { Perímetro } \\
\left(\mathbf{m}^{2}\right)\end{array}$ & DMC & DDC \\
\hline Águas de São Pedro & 1.303 .820 & 4.047 & 644,24 & 0,73 \\
\hline Analândia & 24.055 .141 & 17.386 & $2.767,20$ & 0,95 \\
\hline Araras & 29.723 .479 & 19.326 & $3.076,00$ & 0,72 \\
\hline Capivari & 37.831 .522 & 21.804 & $3.470,27$ & 0,86 \\
\hline Charqueada & 64.744 .858 & 28.524 & $4.539,82$ & 0,97 \\
\hline Conchal & 15.542 .173 & 13.975 & $2.224,29$ & 0,76 \\
\hline Cordeirópolis & 5.413 .959 & 8.248 & $1.312,79$ & 0,67 \\
\hline Corumbataí & 3.067 .773 & 6.209 & 988,21 & 0,70 \\
\hline Elias Fausto & 46.019 .559 & 24.048 & $3.827,43$ & 0,63 \\
\hline Ipeúna & 13.997.318 & 13.262 & $2.110,86$ & 0,96 \\
\hline Iracemápolis & 4.303 .333 & 7.353 & $1.170,41$ & 0,48 \\
\hline Laranjal Paulista & 58.494 .188 & 27.112 & $4.315,12$ & 0,82 \\
\hline Leme & 71.923 .138 & 30.063 & $4.784,87$ & 0,91 \\
\hline Limeira & 100.490 .209 & 35.536 & $5.655,85$ & 0,71 \\
\hline Mombuca & 8.708 .784 & 10.461 & $1.665,00$ & 0,97 \\
\hline Piracicaba & 186.488 .026 & 48.410 & $7.704,80$ & 0,75 \\
\hline Rafard & 5.700 .976 & 8.464 & $1.347,13$ & 0,89 \\
\hline Rio Claro & 57.971 .926 & 26.991 & $4.295,81$ & 0,90 \\
\hline Rio das Pedras & 31.547 .592 & 19.911 & $3.168,98$ & 0,69 \\
\hline Saltinho & 7.990 .899 & 10.020 & $1.594,90$ & 0,95 \\
\hline Santa Gertrudes & 8.517 .750 & 10.346 & $1.646,64$ & 0,89 \\
\hline Santa Maria da Serra & 5.239 .472 & 8.114 & $1.291,46$ & 0,91 \\
\hline São Pedro & 20.632 .956 & 16.102 & $2.562,81$ & 0,44 \\
\hline
\end{tabular}

Fonte: Os autores (2020).

O indicador Distribuição Direcional das Células (DDC), expresso na Tabela 2 pela excentricidade das elipses geradas, permite diferenciar se a distribuição da população está espraiada longitudinalmente ou não: 
quanto mais próximo de 1, mais excêntrica (afilada) é a elipse. Quanto mais próxima de 0 , mais circular. Os resultados mostraram que manchas urbanas menores apresentam maior excentricidade, como Mombuca, Charqueada, Ipeúna e Saltinho. A maior excentricidade das áreas urbanas desses municípios está, geralmente, associada à concentração da população aos vetores rodoviários. Nos municípios citados, a população está distribuída de forma mais longitudinal ou alongada, ou seja, a distribuição segue a influência de algum vetor de ocupação, no caso, as rodovias. Outros municípios como Piracicaba e Limeira parecem menos sujeitos a um vetor, o que pode se justificar por estarem nas áreas de influência de várias rodovias e por outros aspectos tais como características de especulação imobiliária, volume da população, dimensões (porte) da cidade e do município.

Em relação às métricas de fragmentação da mancha urbana, calculadas com base nos polígonos compostos por células urbanas ocupadas contíguas, a Figura 6 apresenta as manchas urbanas de cada município. Nota-se que as cidades de porte médio apresentam uma mancha urbana central com maior volume de população, enquanto ao redor concentram fragmentos de mancha urbana com menor volume. Além disso, cidades pequenas tendem a ter uma maior homogeneização na distribuição do volume de população.

Figura 6 - Manchas Urbanas da Aglomeração Urbana de Piracicaba, 2010.

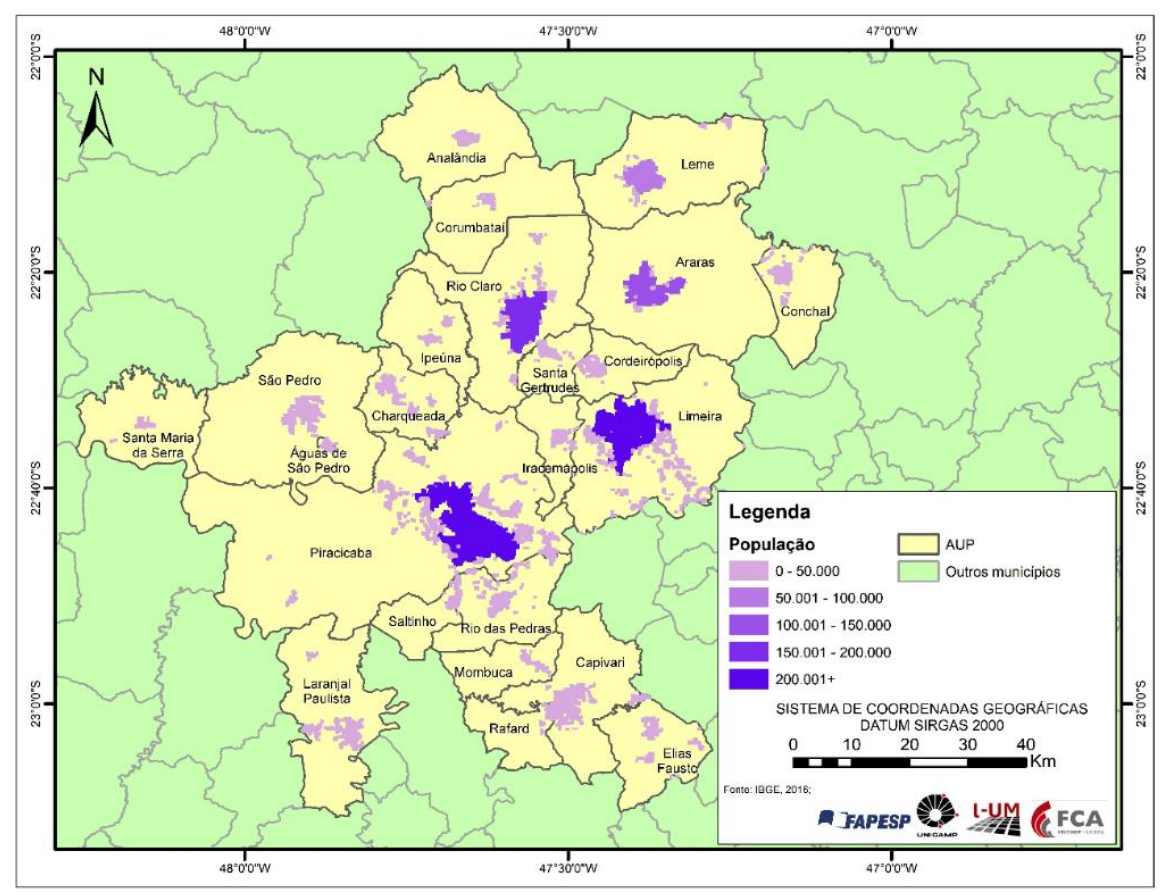

Fonte: Os autores (2020).

A área urbana de Limeira é a que apresenta a maior quantidade de fragmentos, seguido por Piracicaba e Rio Claro (Tabela 3). As três cidades que apresentam as características mais marcantes acerca da dispersão urbana são as que apresentam maior população e maior quantidade de células ocupadas. Entretanto, algumas cidades de porte pequeno, como Rio das Pedras e Capivari, possuem uma quantidade de fragmentos semelhantes às cidades de porte médio, como Rio Claro. 
Tabela 3 - Fragmentação das manchas urbanas da Aglomeração Urbana de Piracicaba, 2010.

\begin{tabular}{|c|c|c|c|c|}
\hline Manchas urbanas & $\begin{array}{l}\text { Área total da } \\
\text { classe }\left(\mathrm{em} \mathrm{m}^{2}\right)\end{array}$ & $\begin{array}{l}\text { Número de } \\
\text { fragmentos }\end{array}$ & $\begin{array}{l}\text { Tamanho médio dos } \\
\text { fragmentos }\left(\mathrm{em} \mathrm{m}^{2}\right)\end{array}$ & $\begin{array}{l}\text { Indicador médio da forma } \\
\text { ponderado pela área }\end{array}$ \\
\hline Águas de São Pedro & 2.203 .860 & 1 & 2.203 .860 & 1,598 \\
\hline Analândia & 5.692 .120 & 3 & 1.897 .370 & 2,462 \\
\hline Araras & 30.480 .700 & 20 & 1.524 .030 & 4,169 \\
\hline Capivari & 19.647 .000 & 44 & 446.522 & 3,298 \\
\hline Charqueada & 10.901 .300 & 28 & 389.332 & 2,739 \\
\hline Conchal & 8.786 .760 & 33 & 266.265 & 2,021 \\
\hline Cordeirópolis & 6.816 .870 & 15 & 454.458 & 2,289 \\
\hline Corumbataí & 2.445 .360 & 14 & 174.668 & 1,808 \\
\hline Elias Fausto & 7.379 .440 & 32 & 230.607 & 2,219 \\
\hline Ipeúna & 3.727 .610 & 10 & 372.761 & 1,706 \\
\hline Iracemápolis & 5.092 .090 & 8 & 636.512 & 1,794 \\
\hline Laranjal Paulista & 12.462 .000 & 41 & 303.952 & 2,912 \\
\hline Leme & 22.940 .900 & 28 & 819.316 & 2,363 \\
\hline Limeira & 76.718 .200 & 161 & 476.511 & 4,057 \\
\hline Mombuca & 2.846 .500 & 17 & 167.441 & 1,503 \\
\hline Piracicaba & 117.008 .000 & 145 & 806.950 & 5,996 \\
\hline Rafard & 2.966 .890 & 5 & 593.379 & 1,958 \\
\hline Rio Claro & 45.704 .400 & 52 & 878.930 & 3,481 \\
\hline Rio das Pedras & 10.102 .200 & 42 & 240.528 & 2,168 \\
\hline Saltinho & 3.847 .980 & 10 & 384.798 & 2,082 \\
\hline Santa Gertrudes & 4.811 .310 & 11 & 437.392 & 2,462 \\
\hline Santa Maria da Serra & 2.443 .200 & 9 & 271.466 & 1,831 \\
\hline São Pedro & 17.670 .000 & 18 & 981.664 & 3,478 \\
\hline
\end{tabular}

Fonte: Os autores (2020).

O tamanho médio dos fragmentos variou de acordo com as áreas urbanas, mostrando diferentes formas de distribuição espacial. Ele não deve ser observado sozinho, pois, cada município apresenta especificidades que caracterizam o tamanho dos seus fragmentos. Já o indicador médio da forma ponderado pela área indica que as áreas urbanas de Piracicaba, Araras e Limeira e Rio Claro possuem forma urbana menos circular que as outras manchas urbanas da AUP. Tais resultados não são diretamente compatíveis com os resultados encontrados com a aplicação dos indicadores de DMC e DDC que indicavam uma mancha urbana mais circular. Isso ocorre devido ao número e o tamanho dos fragmentos das manchas urbanas que influenciam no cálculo de ponderação pela área do indicador, o que representa uma limitação do mesmo se visto isoladamente. Por fim, investigando as possíveis relações entre os indicadores, a Tabela 4 contém as correlações estatísticas lineares (Pearson) entre variáveis selecionadas, e a significância estatística (p-value) de cada correlação.

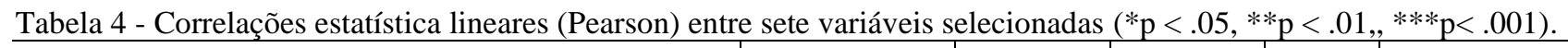

\begin{tabular}{|c|c|c|c|c|c|c|c|}
\hline & & $\begin{array}{l}\text { População } \\
\text { urbana }\end{array}$ & $\begin{array}{c}\text { Células urbanas } \\
\text { ocupadas }\end{array}$ & IMPA & DMC & DDC & $\begin{array}{l}\text { Número de } \\
\text { fragmentos }\end{array}$ \\
\hline \multirow{2}{*}{ População Urbana } & pearson's $r$ & - & & & & & \\
\hline & p-value & - & & & & & \\
\hline \multirow{2}{*}{ Células urbanas ocupadas } & pearson's $r$ & $0.986 * * *$ & - & & & & \\
\hline & p-value & $<.001$ & - & & & & \\
\hline \multirow{2}{*}{ IMPA } & pearson's $r$ & $0.865 * * *$ & $0.903 * * *$ & - & & & \\
\hline & p-value & $<.001$ & $<.001$ & - & & & \\
\hline \multirow{2}{*}{ DCM } & pearson's r & $0.803 * * *$ & $0.833 * * *$ & $0.807 * * *$ & - & & \\
\hline & p-value & $<.001$ & $<.001$ & $<.001$ & - & & \\
\hline \multirow{2}{*}{ DDC } & pearson's $r$ & -0.093 & -0.121 & -0.171 & 0.046 & - & \\
\hline & p-value & 0.672 & 0.583 & 0.435 & 0.833 & - & \\
\hline \multirow{2}{*}{ Número de fragmentos } & pearson's $r$ & $0.901 * * *$ & $0.916^{* * * *}$ & $0.764 * * *$ & $0.819 * * *$ & -0.127 & - \\
\hline & p-value & $<.001$ & $<.001$ & $<.001$ & $<.001$ & 0.563 & - \\
\hline \multirow{2}{*}{ Tamanho dos fragmentos } & pearson's $r$ & 0.108 & 0.096 & 0.186 & -0.030 & -0.082 & -0.129 \\
\hline & p-value & 0.623 & 0.664 & 0.394 & 0.894 & 0.712 & 0.566 \\
\hline
\end{tabular}

Fonte: Os autores (2020). 
O volume da população urbana apresenta forte correlação positiva com o número de células urbanas ocupadas, como se poderia esperar. Ambas as variáveis apresentam forte correlação positiva e significância estatística $(0,001)$ com Distância Média entre as Células $(D M C)$, com o Indicador médio ponderado pela área (IMPA) e com o número de fragmentos. Ou seja, quanto maior o volume da população, maior a desconcentração espacial e maior o número de fragmentos na mancha urbana.

Por outro lado, as correlações das variáveis população urbana e células urbanas ocupadas com a Distribuição Direcional das Células (DDC) e com o tamanho dos fragmentos são muito fracas e sem significância estatística. Ou seja, a população residente e o tamanho da mancha urbana não parecem ter relação com a excentricidade da mancha - correlação negativa - nem com o tamanho dos fragmentos calculados correlação positiva.

As cidades de menor porte parecem ser mais sensíveis aos eixos de ocupação, o que é coerente com a percepção de que o maior volume populacional tem correlação positiva com o número de células ocupadas e as métricas de distribuição. Isto pode ser justificado pela baixa densidade que as cidades brasileiras apresentam, sobretudo as médias e as pequenas, atrelado ao espraiamento da mancha urbana. Ou seja, não há uma concentração espacial com alto volume da população (alta densidade).

O número de fragmentos possui correlação positiva forte com todos os indicadores menos DDC, tal característica pode significar que o crescimento espacial associado à um principal vetor, no caso a uma única rodovia principal, tende a criar uma mancha urbana mais longitudinal e conectada em torno de uma só via. Ao contrário, nas cidades médias que possuem diversas rodovias principais, o número de fragmentos aumenta em todas as direções, de acordo com a disposição das vias.

O número restrito de municípios e a amplitude no número de habitantes e na área urbana dos municípios considerados podem estar dificultando a percepção de relações entre os indicadores - algumas das quais podem não ser lineares, variando conforme o porte da cidade. As possíveis relações entre os indicadores - e a aparente falta de relações entre alguns deles - sugerem a conveniência de novos estudos espaciais que ampliem a base estudada e articulem os resultados quantitativos aos processos de expansão urbana caso a caso.

\section{CONSIDERAÇÕES FINAIS}

As contribuições do artigo se dividem em dois grupos: contribuições da abordagem metodológica ao campo de estudo da forma urbana; contribuições para o estudo da configuração urbana da Aglomeração Urbana de Piracicaba (AUP). Acerca da abordagem adotada, o artigo oferece caminhos para a análise espacial dos fenômenos da dispersão e fragmentação urbana, com resolução espacial maior do que a usualmente considerada, e em unidade territorial de análise propícia aos testes estatísticos, aos estudos comparativos e longitudinais. Ademais e para tal, oferece métricas que permitem analisar quantitativamente dimensões e processos geralmente considerados qualitativamente pela literatura, mas não necessariamente quantificados.

Dessa forma, ao apresentar e testar um conjunto de indicadores espaciais aplicados em dados comumente utilizados para análises demográficas, dados esses de amplo acesso ao público, o artigo explora o uso de grade regulares e o seu potencial para analisar padrões espaciais de distribuição da população, uma frente de estudos ainda pouco usual na literatura sobre as temáticas.

Em relação a AUP, o artigo lançou luz sobre os aspectos espaciais da dispersão e fragmentação urbana neste ente regional recente, tendo sido possível observar que todas as manchas urbanas municipais apresentaram características de dispersão e fragmentação urbana, mesmo que em intensidades diferentes. Tal aspecto contribui para o campo dos estudos urbanos ao apresentar, quantitativamente, os fenômenos em um contexto sócio-espacial não metropolitano.

As correlações testadas apontam para configurações de articulações de determinadas características das manchas urbanas da AUP. Destaque para a correlação entre o volume da população e o número de fragmentos e o volume da população e a sua distribuição espacial, ou seja, as cidades com maior volume de população tendem a ter uma ocupação espacial menos longitudinal e mais circular, mesmo que descontínua. Possivelmente, isso ocorre pelo crescimento desordenado em todas as direções visto em cidades médias brasileiras. Entretanto, tais achados merecem estudos mais aprofundados com uma base de municípios mais ampla. 
Além disso, os resultados indicam que as manchas urbanas das cidades de pequeno porte tendem a apresentar um menor número de fragmentos, porém, espacialmente maiores que tendem a seguir uma principal rodovia. Enquanto isso, as cidades médias tendem a ter um maior número de fragmentos com diferentes tamanhos. A diferenciação entre as cidades médias e pequenas pode estar atrelada a fatores econômicos, históricos e de hierarquia urbana entre as cidades membros da Aglomeração, ou seja, para além de fatores espaciais e do volume da população considerados no artigo, o que é um importante indício para estudos futuros.

Por fim, como as medidas aplicadas para tratar o componente espacial da dispersão e da fragmentação urbana apresentaram-se adequadas para a análise dos fenômenos, sua aplicação e divulgação possibilita outros estudos com maior base de municípios e novos testes estatísticos que avancem no entendimento das relações e dos padrões relevantes aos estudos espaciais dos fenômenos considerados aqui.

Para futuros estudos e desenvolvimento do que foi proposto como abordagem, os indicadores de distribuição são uteis para serem correlacionados com variáveis espaciais e não-espaciais que ajudam a explicar os resultados encontrados nessa pesquisa. Já os indicadores com métricas de paisagem, para além dos fatores já mencionados, são úteis para comparação com outros fenômenos espaciais, sobretudo ambientais, ao possibilitar uma análise da estrutura ambiental da paisagem a partir de características físicas e qual a relação com a mancha urbana.

\section{Agradecimentos}

Os autores agradecem a Fundação de Amparo à Pesquisa do Estado de São Paulo (FAPESP), número do processo 2016/24641-3, pelo apoio financeiro na execução da pesquisa.

\section{Contribuição dos Autores}

Ambos os autores foram responsáveis pela conceptualização do artigo, bem como pela curadoria dos dados, elaboração da metodologia e redação inicial e final do texto apresentado. Destaque para a aplicação e sistematização das análises estatísticas à cargo do Prof. Dr. Álvaro D'Antona, bem como a supervisão da pesquisa de mestrado que resultou neste texto. A organização geral do artigo e a elaboração dos mapas apresentados ficou à cargo do autor principal Ms. José Diego Gobbo Alves.

\section{Conflitos de Interesse}

Os autores declaram que não há conflitos de interesse.

\section{Referências}

AURAMBOUT, J.P; BARRANCO, R.; LAVALLE, C. Towards a Simpler Characterization of Urban Sprawl Across Urban Areas in Europe. Land, v7, 33, 2018. DOI:10.3390/land7010033.

BUENO, M C. D. Grade estatística: uma abordagem para ampliar o potencial analítico de dados censitários. Tese de doutoramento. Programa de Pós-Graduação em Demografia, Universidade Estadual de Campinas, 2014.

BUENO, M C. D.; D’ANTONA, A. O. Avaliação de métodos de desagregação para geração de grades de população. Revista Espinhaço, v. 3, p. 127-137, 2014.

BUENO, M C. D.; D'ANTONA, A. Utilização de grades regulares para análises espaciais intramunicipais de variáveis demográficas: testes para Limeira-SP, 2010. In: XVIII Encontro Nacional de Estudos Populacionais, 2012, Águas de Lindóia. XVIII Encontro Nacional de Estudos Populacionais, 2012.

COELHO, L. L. Dispersão, fragmentação e paisagem: relações entre dinâmicas naturais e urbanas no vetor oeste da Região Metropolitana de São Paulo. Tese de doutoramento. Programa de PósGraduação em Arquitetura e Urbanismo. Universidade de São Paulo, 2015. 
COELHO, L. L. Os conceitos de dispersão e fragmentação urbana sob a abordagem da paisagem. In: Anais do IV Encontro da Associação Nacional de Pesquisa e Pós-Graduação em Arquitetura e Urbanismo, Porto Alegre, 2016.

CÔRTES, J. C. Ciclo de vida familiar e distribuição populacional na dinâmica do desmatamento e uso da terra na Amazônia Paraense. Tese. Doutorado em Demografia. Universidade Estadual de Campinas, 2017.

CUNHA, J.M.P.; et al. A mobilidade pendular na macrometrópole paulista: diferenciação e complementariedade socioespacial. Cadernos Metrópole, 15 (30), 2013.

D'ANTONA, A. O.; BUENO, M. C. DISTRIBUIÇÃO DA POPULAÇÃO EDISPERSÃO URBANA NO ESTADODE SÃO PAULO, 2010. In OJIMA, R.; MARANDOLA Jr, E. (Org.) Dispersão urbana e mobilidade populacional - Teoria, Método e Evidências. Blücher. 2015a.

D’ANTONA, A. O.; BUENO, M. C. Grades estatísticas no estudo da dispersão urbana- superação dos limites das unidades administrativas e operacionais censitárias. In OJIMA, R.; MARANDOLA Jr, E. (Org.) Dispersão urbana e mobilidade populacional - Teoria, Método e Evidências. Blücher. 2015b.

D'ANTONA, A. O.; BUENO, M. C. D.; DAGNINO, R. S. Estimativa da população em unidades de conservação na Amazônia Legal brasileira - uma aplicação de grades regulares a partir da Contagem 2007. Revista Brasileira de estudos populacionais., Rio de Janeiro, v. 30, n. 2, p. 401-428, jul./dez. 2013.

DEPARTAMENTO NACIONAL DE INFRAESTRUTURA DE TRANSPORTES (DNIT). Atlas e Mapas.Base Cartográfica
http://www.dnit.gov.br/download/mapasmultimodais/shapefiles/>

EIGENHEER, D. M.; SOMEKH, N. Formas avançadas de dispersão urbana no vetor noroeste paulista: eixo São Paulo-Campinas. Cad. Metrop., São Paulo, v. 19, n. 40, pp. 777-797, set/dez 2017.

EMPRESA PAULISTA DE PLANEJAMENTO METROPOLITANO (EMPLASA). Aglomeração urbana de Piracicaba. 2018. Disponível em <https://www.emplasa.sp.gov.br/AUP>. Acessado em: 01/07/2019.

EMPRESA PAULISTA DE PLANEJAMENTO METROPOLITANO (EMPLASA). Panorama Regional PDUI. 2017. Disponível em: < https://www.pdui.sp.gov.br/piracicaba/?page_id=755> Acessado em: $01 / 07 / 2019$

ESBAH, $\mathrm{H}$ et al. Understanding Urban Growth Patterns: A Landscape Ecology Point of view. In: International Society For Photogrammetry And Remote Sensing, 2012. Disponível em: <https://www.isprs.org/proceedings/XXXVI/8-W27/esbah01.pdf>.

ENVIRONMENTAL SYSTEMS RESEARCH INSTITUTE (ESRI). ARCMAP. Directional Distribution (Standard Deviational Ellipse). 2019a. $\quad$ Disponível em http://desktop.arcgis.com/en/arcmap/10.3/tools/spatial-statistics-toolbox/directional-distribution.htm S.a Acessado em: 01/07/2019

ENVIRONMENTAL SYSTEMS RESEARCH INSTITUTE (ESRI). ARCMAP. Standard Distance. 2019b. Disponível em http://desktop.arcgis.com/en/arcmap/10.3/tools/spatial-statistics-toolbox/standarddistance.htm. Acessado em: 01/07/2019

EWING, R. et al. Mensuring sprawl and its impact. Technical Report, v. 1, Smart Growth America, Washington, 2002.

FRENKEL, A.; ASHKENAZI, M. Measuring urban sprawl: how can we deal with it. Environment and Planning B: Planning and Design, V.35, 2008.

GALSTER, G. et al. Wrestling sprawl to the Ground: Defining and Measuring an Elsive Concept. Housing Policy Debate, v. 12, Issue 4, 2001.

GONÇALVES, A. R. Indicadores de Dispersão urbana. Dissertação de Mestrado. Universidade Federa do Rio Grande do Sul, Programa de Pós-Graduação em Planejamento Urbano e Regional, Porto Alegre, 2011. 113p

GONÇALVES, A. R.; KRAFTA, R. Indicador de dispersão urbana baseado em medida de acessibilidade ponderada. Revista Brasileira de Cartografia, v. 68, n. 4, 18 abr. 2016.

INSTITUTO BRASILEIRO DE GEOGRAFIA E ESTATÍSTICA (IBGE). Base cartográfica: Grade estatística. 2016 Disponível em: <ftp://geoftp.ibge.gov.br/recortes_para_fins_estatisticos/grade_estatistica/censo_2010/>. Acessado em: 20/08/2018.

IWAMA, A. Y. et al. RISCOS GEOTÉCNICOS E VULNERABILIDADES: UMA ABORDAGEM 
UTILIZANDO GRADE REGULAR ESTATÃ• STICA EM UMA ZONA COSTEIRA DO BRASIL.

Revista Brasileira de Cartografia, v. 68, n. 5, 20 jun. 2016.

LIMONAD, E. Urbanização dispersa mais uma forma de expansão urbana? Formação (Presidente Prudente), v. 1, p. 31-45, 2007

LIU, Y. et al. Impacts of land finance on urban sprawl in China: The case of Chongqing. Land Use Policy 72 (2018) 420-432

LLOYD, C.D. et al. Exploring the utility of grids for analysing long term population change. Computers, Environment and Urban Systems, Volume 66, Novembr0, 2017

MANCINI, G. A. Avaliação dos custos da urbanização dispersa no Distrito Federal. Universidade de Brasília. Programa de Pesquisa e Pós-Graduação em Arquitetura e Urbanismo.200

MARANDOLA JUNIOR, E. J.; MAGOSSO, C. MOBILIDADE ESPACIAL DA POPULAÇÃO E REGIONALIZAÇÃO NA REGIÃO DE LIMEIRA (SP). In: OJIMA, R; MARANDOLA Jr., E. Dispersão Urbana e Mobilidade Populacional. São Paulo: Blucher, 2016.

MEADOWS, D.H. So what can we do - really do - about sprawl. In Sprawl Articles, Sierra Club, 1999.

NANDIN, C. L. C. Urbanização, dispersão e morfologia urbana: o eixo noroeste da Região Metropolitana de Campinas. Dissertação de Mestrado. Pontifícia Universidade Católica de Campinas, Programa de Pós-Graduação em Urbanismo, Campinas, 2013.

NASCIMENTO JÚNIOR, L. Urbanização e cidade dispersa: implicações da produção do espaço urbano no Brasil, em Moçambique e na Austrália. Geousp - Espaço e Tempo (Online), v. 21, n. 2, p. 550-569, agosto. 2017. ISSN 2179-0892

OJIMA, R. Análise comparativa da dispersão urbana nas aglomerações urbanas brasileiras: elementos teóricos e metodológicos para o planejamento urbano e ambiental. Tese de doutoramento. Universidade Estadual de Campinas, Programa de Pós-Graduação em Demografia, Campinas - SP, 2007. 166p

PEDRO, A. A.; QUEIROZ FILHO, A. P. DE. AVALIAÇÃO DA GRADE ESTATÍTICA EM AGLOMERADOS SUBNORMAIS: ESTUDO DE CASO DA SUBPREFEITURA DE SÃO MATEUS - SP. Revista Brasileira de Cartografia, v. 69, n. 2, 22 fev. 2017.

REIS FILHO, N. G. Notas sobre urbanização dispersa e novas formas de tecido urbano. São Paulo: Via das Artes, 2006

SÃO PAULO. Lei Complementar Estadual No 1.178 de 26 de junho de 2012. São Paulo: SP, 2012

SCHUTZER, J. G. Dispersão urbana e apropriação do relevo na macrometrópole de São Paulo. 2012. Tese (Doutorado em Geografia Física) - Faculdade de Filosofia, Letras e Ciências Humanas, Universidade de São Paulo, São Paulo, 2012. doi:10.11606/T.8.2012.tde-12042013-104250. Acesso em: 2020-01-08.

SPOSITO, M. E. B. Urbanização difusa e cidades dispersas: perspectivas espaço-temporais contemporâneas. In: REIS, Nestor G. (Org.). Sobre a dispersão urbana. São Paulo: Via das Artes/FAUUSP, 2009. p. $38-54$

TAGIL, S.; GORMUS, S.; CENGIZ, S. The Relationship of Urban Expansion, Landscape Patterns and Ecological Processes in Denizli, Turkey. Journal of the Indian Society of Remote Sensing. v.46, p. 1-12. 2018

TRENTIN, G. Dimensão fractal, dinâmica espacial e padrões de fragmentação urbana de cidades médias do estado de São Paulo. Tese de doutoramento. Universidade Estadual de Campinas, Programa de PósGraduação em Geografia, Campinas, 2012. 238p

VALADARES, A. A. O Gigante Invisível: Território e População Rural para Além Das Convenções Oficiais. Instituto de Pesquisa Econômica Aplicada. Texto para discussão 1942, 2014.

ZHOU, Y.; VARQUEZ, A.C.G.; KANDA M. High-resolution global urban growth projection based on multiple applications of the SLEUTH urban growth model. Sci Data. Abril 18;6(1):34, 2019. doi: 10.1038/s41597-019-0048-z. 


\section{Biografia do autor principal}

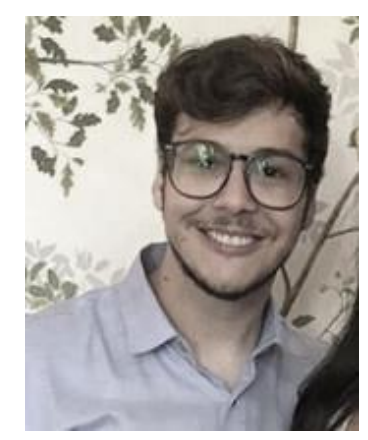

José Diego Gobbo Alves, Piracicaba (São Paulo - Brasil), 1994. Geógrafo pela Universidade Estadual Paulista (UNESP), Mestre em Ciências Humanas e Sociais Aplicadas pela Universidade Estadual de Campinas (Unicamp) e, atualmente, doutorando em Ambiente e Sociedade também pela Unicamp. Bolsista da Fundação de Amparo à Pesquisa do estado de São Paulo (FAPESP) na graduação e no mestrado. Tem interesse nas áreas de Mudanças no uso e cobertura da terra; População e Ambiente; Distribuição espacial da população; Ambiente e Sociedade; Planejamento Urbano e Regional; Mobilidade Urbana e Sustentável; Urbanização e Política Urbana. devido crédito pela criação original. 